\title{
YTHDF3 facilitates triple-negative breast cancer progression and metastasis by stabilizing ZEB1 mRNA in an $\mathbf{m}^{6} \mathrm{~A}$-dependent manner
}

\author{
Yuxiang Lin ${ }^{1,2,3 \#}$, Xuan Jin ${ }^{1,2,3 \#}$, Qian Nie ${ }^{1,2,3 \#}$, Minyan Chen ${ }^{1,2,3 \#}$, Wenhui Guo ${ }^{1,2,3}$, Lili Chen ${ }^{1,2,3}$, \\ Yan $\mathrm{Li}^{1,2,3}$, Xiaobin Chen ${ }^{1,2,3}$, Wenzhe Zhang ${ }^{1,2,3}$, Hanxi Chen ${ }^{1,2,3}$, Meichen Jiang ${ }^{4}$, Han Xiao ${ }^{4}$, Jie Zhang ${ }^{1,2,3}$, \\ Fangmeng Fu ${ }^{1,2,3}$, Chuan Wang ${ }^{1,2,3}$ \\ ${ }^{1}$ Department of Breast Surgery, Fujian Medical University Union Hospital, Fuzhou, China; ${ }^{2}$ Department of General Surgery, Fujian Medical \\ University Union Hospital, Fuzhou, China; ${ }^{3}$ Breast Cancer Institute, Fujian Medical University, Fuzhou, China; ${ }^{4}$ Department of Pathology, Fujian \\ Medical University Union Hospital, Fuzhou, China \\ Contributions: (I) Conception and design: Y Lin, F Fu, C Wang; (II) Administrative support: Y Lin, J Zhang; (III) Provision of study materials or \\ patients: X Jin, Q Nie, W Zhang, H Chen; (IV) Collection and assembly of data: W Guo, L Chen, M Chen; (V) Data analysis and interpretation: H \\ Xiao, Y Li, X Chen, M Jiang; (VI) Manuscript writing: All authors; (VII) Final approval of manuscript: All authors. \\ \#These authors contributed equally to this work. \\ Correspondence to: Jie Zhang, PhD; Fangmeng Fu, PhD; Chuan Wang, PhD. Department of Breast Surgery, Fujian Medical University Union Hospital, \\ No. 29, Xin Quan Road, Gulou District, Fuzhou 350001, China. Email: zj1979@hotmail.com; ffm@fjmu.edu.cn; chuanwang1968@outlook.com.
}

Background: The Y'TH domain family protein 3 (Y'THDF3) is an important N6-methyladenosine $\left(\mathrm{m}^{6} \mathrm{~A}\right)$ reader which is involved in multiple cancers. However, the biological role and mechanisms of action for YTHDF3 in triple-negative breast cancer (TNBC) remains to be elucidated.

Methods: The expression of YTHDF3 in TNBC tissues was evaluated using The Cancer Genome Atlas (TCGA) database, BC-GenExMiner, and immunohistochemistry (IHC) staining. Cell migration, invasion, and epithelial-mesenchymal transition (EMT) were validated by wound healing assays, transwell assays, and Western blot (WB) analyses. The association between YTHDF3 and zinc finger E-box-binding homeobox 1 (ZEB1) was confirmed by Pearson correlation analysis. RNA-binding protein immunoprecipitation (RIP) assays and mRNA actinomycin stability analyses were applied to confirm whether YTHDF3 could interact with ZEB1in an $\mathrm{m}^{6} \mathrm{~A}$-dependent manner.

Results: The expression of YTHDF3 was correlated with poorer disease-free survival (DFS) and overall survival (OS) in TNBC patients. Functional experiments indicated that YTHDF3 positively regulated cell migration, invasion, and EMT in TNBC cells. Moreover, ZEB1 was identified as a key downstream target for YTHDF3 and YTHDF3 could enhance ZEB1 mRNA stability in an $\mathrm{m}^{6} \mathrm{~A}$-dependent manner. Inhibition of YTHDF3 reduced migration, invasion, and EMT, all of which were reversed by rescue experiments overexpressing ZEB1.

Conclusions: The findings herein confirmed that the YTHDF3/ZEB1 axis plays an important role in the progression and metastasis of TNBC. YTHDF3 is a promising prognosis biomarker and potential therapeutic target for patients with TNBC.

Keywords: Triple-negative breast cancer (TNBC); metastasis; epithelial-mesenchymal transition (EMT); YTH domain family 3 (YTHDF3); zinc finger E-box-binding homeobox 1 (ZEB1)

Submitted Nov 30, 2021. Accepted for publication Jan 14, 2022.

doi: 10.21037/atm-21-6857

View this article at: https://dx.doi.org/10.21037/atm-21-6857

^ ORCID: 0000-0002-3963-3468. 


\section{Introduction}

Breast cancer (BC) is now the most common malignant tumor for women worldwide (1). Triple-negative breast cancer (TNBC) is one of the most aggressive BC subtypes, characterized by the lack of estrogen receptor (ER), progesterone receptor (PR), and human epidermal growth factor receptor 2 (HER-2) expression (2). Although TNBC only accounts for about $15-20 \%$ of all BC cases, treatment response is generally poor, with high metastatic potential and unfavorable prognosis (3). To date, there is no effective targeted therapy for TNBC other than surgery and conventional chemotherapy $(2,4)$. Therefore, novel prognostic biomarkers and molecular therapeutic targets for TNBC patients are urgently needed.

N6-methyladenosine $\left(\mathrm{m}^{6} \mathrm{~A}\right)$ is the most abundant internal modification of RNA in eukaryotic cells and regulates almost all important biological processes by mRNA splicing, translation, and stability (5-8). There are three types of $\mathrm{m}^{6} \mathrm{~A}$ regulators which includes $\mathrm{m}^{6} \mathrm{~A}$ "writers" $\left(\mathrm{m}^{6} \mathrm{~A}\right.$ methyltransferases including METTL3, METTL14, and WTAP), "erasers" ( $\mathrm{m}^{6} \mathrm{~A}$ demethylases including FTO and $\mathrm{ALKBH} 5)$, and "readers" ( $\mathrm{m}^{6} \mathrm{~A}$ binding proteins including YTH domain family protein 1-3, YTH domain containing 1-2 and HNRNPA2B1). Increasing evidence have indicated that the $\mathrm{m}^{6} \mathrm{~A}$ regulators are involved in multiple cancers and play vital roles in tumor cell proliferation and metastasis $(9,10)$. Several m6A regulators, such as METTL3, FTO and HNRNPA2B1, have been identified to promote the malignant progression and therapeutic response of $\mathrm{BC}$ (11-14). YTH domain family protein 3 (YTHDF3) is an important reader as it plays a significant role in modulating translation of m6A-modified mRNAs and affects methylated mRNA decay in an $\mathrm{m}^{6} \mathrm{~A}$ methylation-dependent manner $(15,16)$. YTHDF3 has been shown to contribute to the progression of several types of tumors, including gastric and colorectal cancer $(17,18)$. In patients with $\mathrm{BC}$, YTHDF3 has been associated with brain metastasis and poor prognosis $(19,20)$. However, to date, there have been no studies specifically describing the function of YTHDF3 in TNBC. Our previous proteomic study indicated that YTHDF3 was highly expressed in TNBC tumor tissues with more advanced grade (21). Therefore, this study was conducted to validate the expression and function of YTHDF3 in TNBC.

The present study showed that YTHDF3 expression was closely associated with lymph node metastasis and higher histological grade in patients with TNBC. YTHDF3 was also indicated to be an independent prognostic biomarker for TNBC patients and knockdown of YTHDF3 inhibited the migration, invasion, and epithelial-mesenchymal transition (EMT) in TNBC cells. Furthermore, the EMT transcriptional factor zinc finger E-box-binding homeobox 1 (ZEB1) was identified as a key target of YTHDF3 and YTHDF3 could enhance ZEB1 mRNA stability in an $\mathrm{m}^{6} \mathrm{~A}-$ dependent manner. Taken together, these findings offer insights into novel therapeutic targets in patients with TNBC.

We present the following article in accordance with the MDAR reporting checklist (available at https://atm. amegroups.com/article/view/10.21037/atm-21-6857/rc).

\section{Methods}

\section{Gene expression profiling analysis}

The microarray datasets of TNBC patients were extracted from The Cancer Genome Atlas (TCGA) database (https:// portal.gdc.cancer.gov/). BC-GenExMiner (http://bcgenex. ico.unicancer.fr/) is a statistical mining tool with published annotated BC transcriptomic data (22). The prognostic module was used to analyze the prognostic effects of YTHDF3 in TNBC.

\section{TNBC tumor samples}

A total of 224 TNBC tissue specimens were selected from female patients (aged 27-72 years) undergoing surgery in the Fujian Medical University Union Hospital between June 2013 and June 2018. All patients received radical surgery and had at least six cycles of adjuvant chemotherapy. Disease-free survival (DFS) was calculated as the time from the date of diagnosis to the date of clinical relapse (with histopathology confirmation or radiological evidence of tumor recurrence). Overall survival (OS) was calculated as the time from the date of diagnosis until death from any cause. The last follow-up time was July 1, 2021. All patients agreed to the use of their specimens and signed the informed consent forms. This study was approved by the Research Ethics Committee of Fujian Medical University Union Hospital (No. 2021KJCX039). All procedures performed in this study involving human participants were in accordance with the Declaration of Helsinki (as revised in 2013). 


\section{Cell culture and transfection}

The human TNBC cell lines MDA-MB-231, BT-549, HCC1937, and Hs578T were purchased from the Cell Bank of Type Culture Collection of The Chinese Academy of Sciences. Cell were cultured in Dulbecco's Modified Eagle Medium (DMEM; HyClone, Logan, USA), supplemented with $10 \%$ fetal bovine serum (FBS; Gibco, Carlsbad, USA) and $1 \%$ penicillin and streptomycin solution and incubated at $37{ }^{\circ} \mathrm{C}$ and $5 \% \mathrm{CO}_{2}$. The short hairpin RNA (shRNA) targeting YTHDF3 was subcloned into the GV493 lentiviral shRNA vector (Genechem, Shanghai, China). For overexpression of ZEB1, the construct was generated by subcloning PCR amplified full-length human ZEB1 cDNA into the vector. The constructed lentiviral vectors were packaged into viruses in $293 \mathrm{~T}$ cells which were then harvested and the concentrated viruses were added to TNBC cells and cultured for 72 hours. Gene sequences are provided in Table S1.

\section{Quantitative real time-polymerase chain reaction ( $q R T$ - PCR)}

Total RNA was extracted using TRIzol reagent (Invitrogen, Carlsbad, USA) and the complementary DNA was synthesized by PrimeScript RT Master Mix (Takara, Dalian, China) according to the manufacturer's instructions. The SYBR Green kit (Takara, Dalian, China) was used for qRT-PCR (model 7500 Real-Time PCR System; Applied Biosystems) according to the manufacturer's protocol. The glyceraldehyde 3-phosphate dehydrogenase (GAPDH) gene was used for normalization of data. The fold change in gene expression was calculated using the $2^{-\Delta \Delta \mathrm{ct}}$ method with three independent replicates of all biological samples. The primer sequences are listed in Table S1.

\section{Wound healing migration assay}

Cells were seeded onto 96-well plates and cultured overnight. A line wound was made by scraping a $10 \mu \mathrm{L}$ pipette tip across the confluent cell layer. The floating cells were washed three times with phosphate buffered saline (PBS) and the serum-free medium was applied to maintain the cells. Cell images were captured at 0 and 24 hours with an inverted light microscope.

\section{Transwell invasion assays}

Cells were washed with PBS and suspended in DMEM without FBS at $1 \times 10^{5}$ cells $/ \mathrm{mL}$. The upper chamber of the Transwell (Corning, New York, USA) was precoated with Matrigel (BD Biosciences, San Diego, USA) and filled with $100 \mu \mathrm{L}$ of cell suspension. The lower chamber was filled with $600 \mu \mathrm{L}$ DMEM supplemented with $30 \%$ FBS. After incubation for 24 hours at $37{ }^{\circ} \mathrm{C}$, cells were fixed with $4 \%$ paraformaldehyde for 30 minute and stained with $0.5 \%$ crystal violet for 5 minutes at room temperature. The chamber was then washed with PBS solution and the cells on the surface of the chamber were wiped off with cotton swabs. The images of the stained cells on the lower side were captured (×200 magnification).

\section{WB analysis}

Total protein was extracted using RIPA lysis buffer (Beyotime, Shanghai, China) and the concentration was determined with the BCA Protein Assay Kits (Beyotime, Shanghai, China). A total of $20 \mu \mathrm{g}$ of protein was loaded for sodium dodecyl sulphate polyacrylamide gel electrophoresis (SDS-PAGE). Samples were transferred to polyvinylidene fluoride (PVDF) membranes and incubated with the following primary antibodies: anti-YTHDF3 (ab220161, Abcam, Cambridge, UK), anti-ZEB 1 (21544-1-AP, Proteintech, Wuhan, China), anti-E-cadherin (20874-1-AP, Proteintech, Wuhan, China), anti-N-cadherin (22018-1-AP, Proteintech, Wuhan, China), anti-vimentin (10366-1-AP, Proteintech, Wuhan, China), and anti-GAPDH (ab181602, Abcam, Cambridge, UK). Membranes were then incubated with a secondary horseradish peroxidase-conjugated antibody and visualized with the ECL reagent (Thermo Fisher Scientific, Waltham, USA).

\section{Immunohistochemistry (IHC) staining}

IHC staining analysis was performed on paraffin-embedded tissues to assess the protein expression of YTHDF3 in TNBC tissues. Slides were incubated with anti-YTHDF3 (1:800; ab220161, Abcam, Cambridge, UK) and anti-ZEB1 (1:800, 21544-1-AP, Proteintech, Wuhan, China) antibody according to the standard procedure. The IHC staining scores of YTHDF3 were evaluated by 2 independent 
pathologists blinded to the corresponding patients. The percentage of stained positive cells was scored from 1 to 4 as followed: 1 point for $0-25 \%$ of cells stained; 2 points for $26-50 \%$ of cells stained; 3 points for $51-75 \%$ of cells stained; and 4 points for $75-100 \%$ of cells stained. The staining intensity score was calculated from 0 to 3 as follows: 0 points indicates no staining; 1 point indicates weak staining; 2 points indicates moderate staining; and 3 points indicates strong staining. The percentage of positive tumor cells and the staining intensity were multiplied to produce a weighted score for each patient. A score of 8-12 was defined as high expression level and a score of $0-7$ was defined as low expression.

\section{RNA-binding protein immunoprecipitation (RIP) assay}

The RIP assay was conducted with Magna RIP Kits (Millipore, Billerica, USA) according to the manufacturer's instructions. The antibodies used in this study were antiYTHDF3 (ab220161, Abcam, Cambridge, UK), antim6a (202003, Synaptic Systems, Goettingen, Germany), and normal IgG. The RNA complexes were extracted by proteinase $\mathrm{K}$ and phenol/chloroform/isoamyl alcohol, and amplified by qRT-PCR.

\section{RNA stability assay}

To detect the ZEB1 mRNA stability, the cells were treated with actinomycin $(6 \mathrm{mg} / \mathrm{mL})$ for $0,2,4,6$, and 8 hours. The relative RNA level was measured by qRT-PCR.

\section{Statistical analysis}

Each experiment was repeated 3 times and presented as the means \pm standard deviation (SD) in this study. The student's $t$-test was used to compare variables between two groups. The Chi-square test was used to examine the clinicopathological characteristics between patients with high and low YTHDF3 expression. The correlation between the expression levels of YTHDF3 and ZEB1 was evaluated by Spearman rank correlation coefficients. Survival curves were plotted by the Kaplan-Meier method and analyzed by log-rank tests. Cox proportional hazard regression model was performed for univariate and multivariate survival analysis. A two-sided $\mathrm{P}$ value of less than 0.05 was considered statistically significant. All statistical analyses were performed using the SPSS 20.0 software (IBM, USA) and GraphPad Prism 7.0 software (USA).

\section{Results}

\section{YTHDF3 is correlated with clinicopathological features and predicts poor prognosis in TNBC patients}

To detect YTHDF3 expression in TNBC tumor tissues, data mining was performed and the YTHDF3 mRNA profiles from the publicly available TCGA database and BC-GenExMiner were analyzed. The mRNA expression of YTHDF3 was shown to be associated with the DFS for patients with TNBC [hazard ratio $(\mathrm{HR})=6.79$, $95 \%$ confidence interval $(\mathrm{CI}): 1.13$ to $7.01, \mathrm{P}=0.048$; and $\mathrm{HR}=1.27,95 \% \mathrm{CI}: 1.09$ to 1.48 ; Figure $1 \mathrm{~A}, 1 \mathrm{~B}]$. Immunohistochemical (IHC) staining was conducted on the 224 TNBC patient specimens and the YTHDF3 protein expression level was scored based on the staining intensity and percentage of positive tumor cells (Figure 1C). Consistent with our previous proteomic study (16), high YTHDF3 expression was correlated with more advanced histological grade $(\mathrm{P}=0.014$; Table 1). In addition, patients with elevated expression of YTHDF3 had a greater probability of lymph node metastasis $(\mathrm{P}=0.028$; Table 1$)$. Moreover, Kaplan-Meier survival analysis showed that high YTHDF3 expression exhibited a significantly poorer DFS and OS compared to those patients with low YTHDF3 expression (Figure 1D,1E). Multivariate Cox analysis revealed that YTHDF3 expression is an independent prognostic factor for both DFS and OS (HR=1.93, 95\% CI: 1.14 to $3.28, \mathrm{P}=0.015$; and $\mathrm{HR}=1.99,95 \% \mathrm{CI}: 1.02$ to 3.86 , $\mathrm{P}=0.044$, respectively; Table 2). In summary, these results indicated that high expression of YTHDF3 is a risk factor and YTHDF3 may be a potential prognostic biomarker for patients with TNBC.

\section{Suppressing YTHDF3 inbibits migration, invasion, and endothelial-mesenchymal transition in TNBC cells}

The protein expression of YTHDF3 was assessed in 4 TNBC cell lines (Figure $2 A$ ). The MDA-MB-231 and BT-549 cell lines showed higher YTHDF3 expression and were selected for further studies. Three shRNAs were designed and synthesized to suppress YTHDF3 expression in these cell lines (Figure 2B). The shRNAs that exhibited higher interference efficiency was validated by $\mathrm{WB}$ and chosen for subsequent experiments (Figure 2C). The wound healing assay demonstrated that YTHDF3 inhibition significantly reduced the migration potential of both cell lines (Figure 2D). In the transwell assays, a notably decrease in invasion ability was observed when 
A

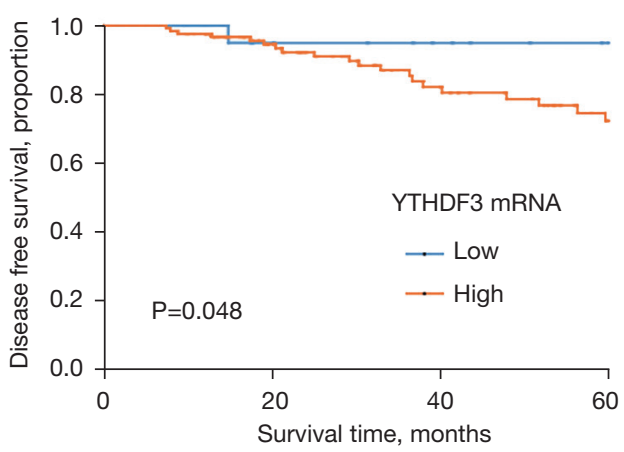

B

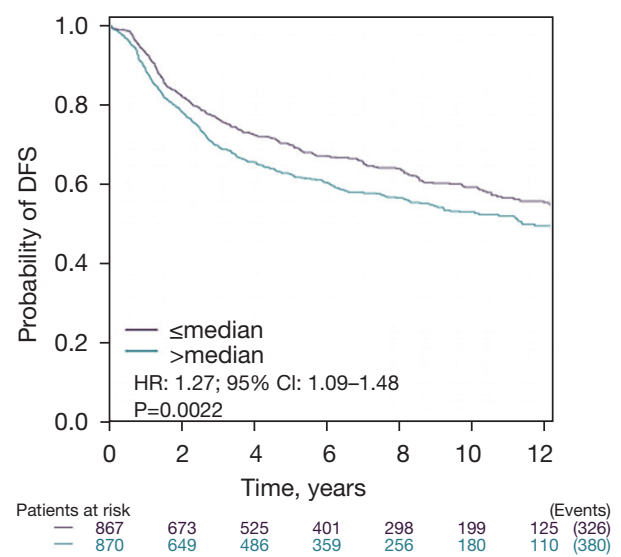

C
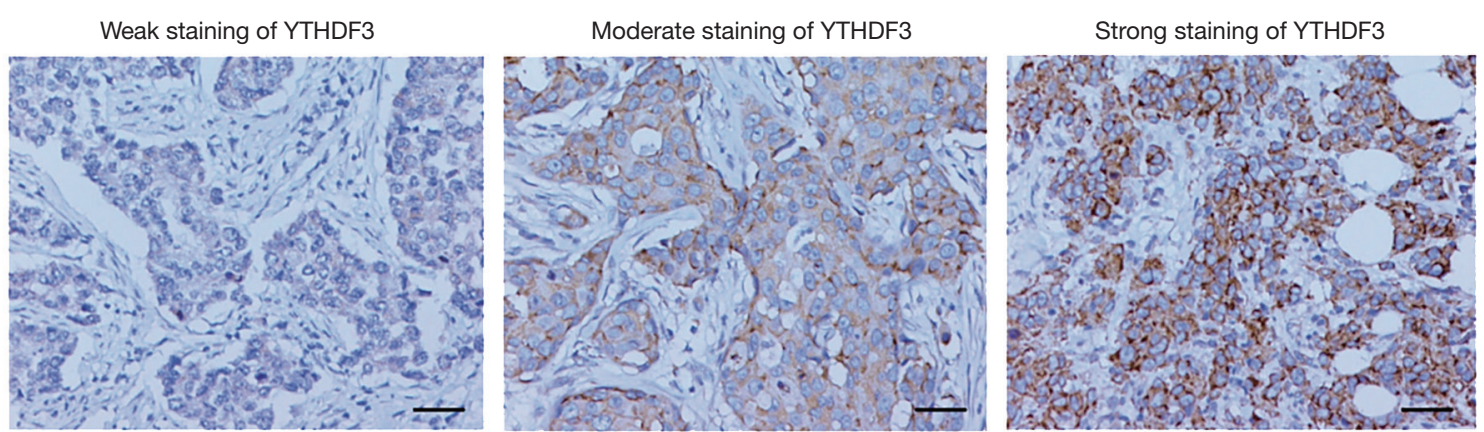

D

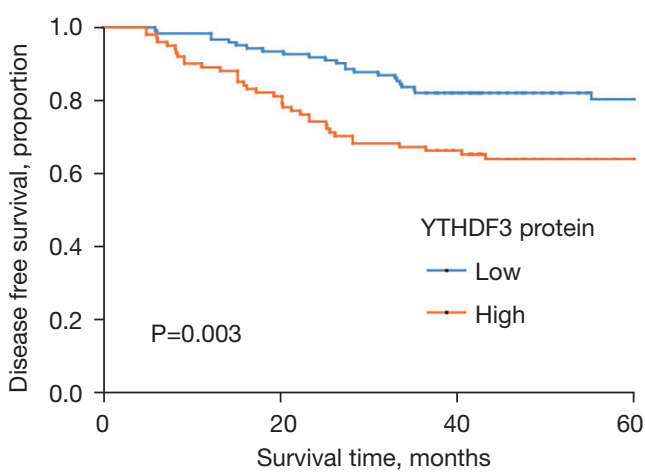

$\mathrm{E}$

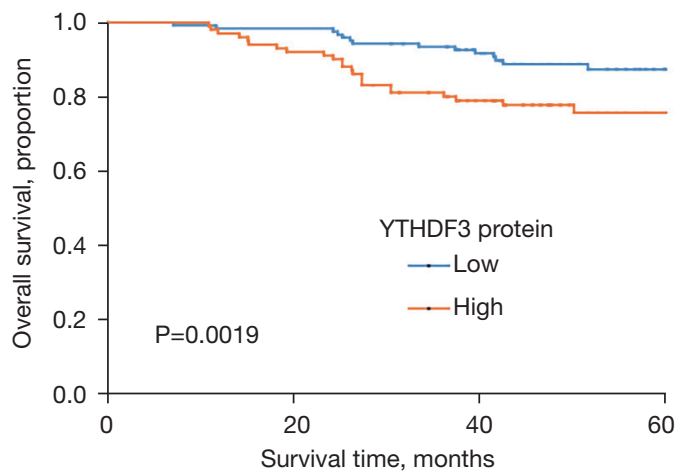

Figure 1 YTHDF3 expression is correlated with poor prognosis in TNBC patients. (A,B) Kaplan-Meier analysis of the association between DFS and YTHDF3 mRNA expression in TNBC patients using TCGA database and BC-GenExMiner. (C) Representative IHC images of weak, moderate, and strong staining of YTHDF3 in TNBC tumor tissues ( $\times 200$ magnification, scale bar: $50 \mu \mathrm{m})$. (D,E) Kaplan-Meier analysis of the association between DFS and OS with YTHDF3 protein expression in TNBC patients using the IHC method. YTHDF3, the YTH domain family 3; TNBC, triple-negative breast cancer; DFS, disease-free survival; TCGA, The Cancer Genome Atlas; IHC, immunohistochemistry; OS, overall survival.

YTHDF3 expression was knocked down (Figure 2E). In addition, WB ting was performed to assess the expression levels of several EMT markers related to cell migration and invasion. Knockdown of YTHDF3 upregulated the expression of E-cadherin and downregulated the expression of $\mathrm{N}$-cadherin and vimentin compared with the shRNA negative control group (shCtrl) (Figure 2F). Taken together, these findings demonstrated that YTHDF3 promotes the migration, invasion, and EMT ability in TNBC cells. 
Table 1 Associations of YTHDF3 expression with clinicopathological characteristics for triple-negative breast cancer patients

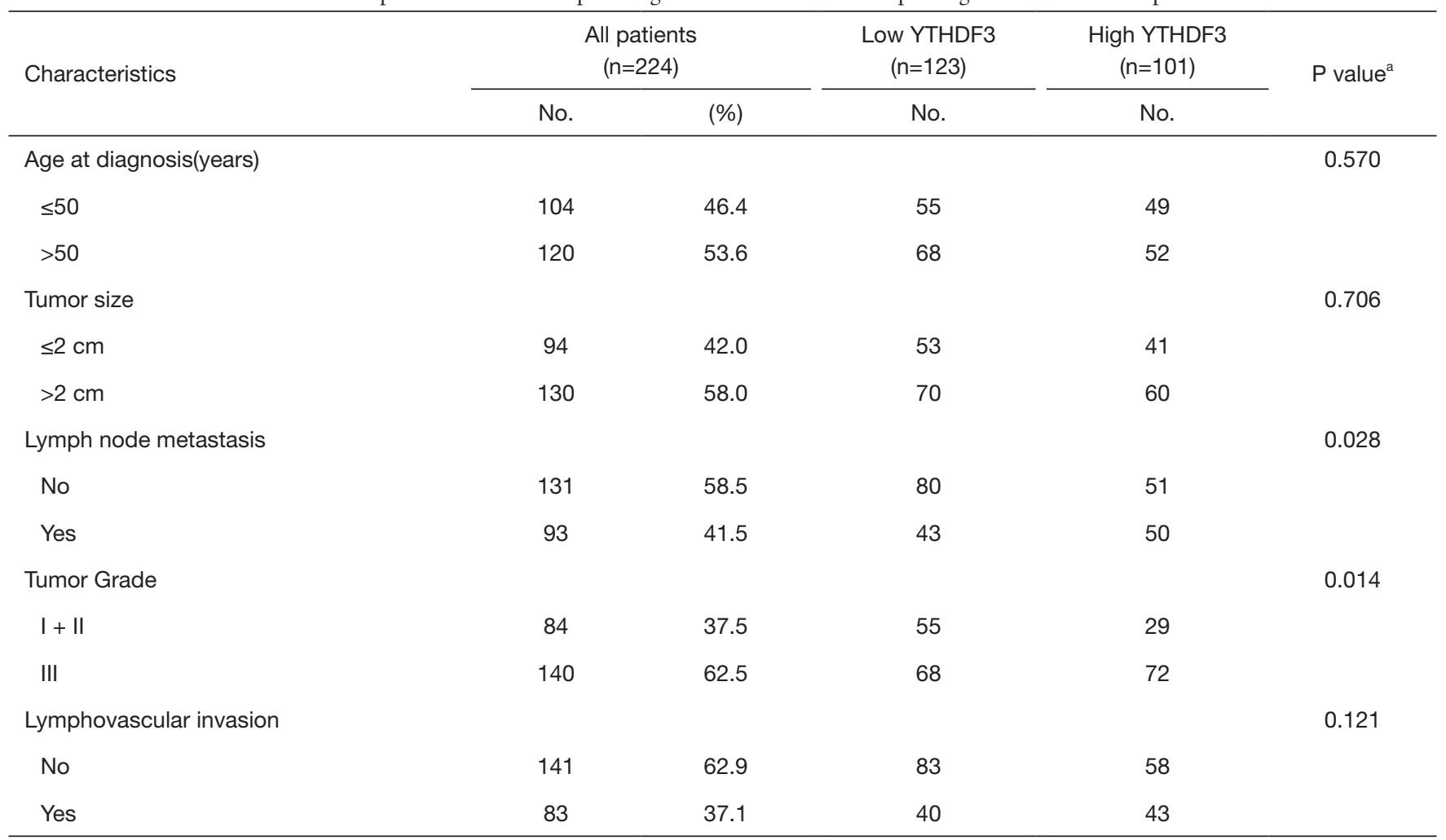

${ }^{a}$, the $\mathrm{P}$ value was calculated among all groups by the Chi-square test. YTHDF3, the YTH domain family 3.

\section{YTHDF3 regulates ZEB1 expression in TNBC}

EMT is regulated by a complicated network. It is well known that several EMT transcriptional factors (EMT-TFs), namely SNAIL, TWIST, and members of the ZEB family, play vital roles in metastasis and drug resistance in cancer (23-27). To explore the underlying mechanisms of YTHDF3 in TNBC, the correlation between the mRNA expression levels of YTHDF3 and EMT-TFs in TNBC samples was examined using the TCGA database (Figure $3 A-F$ ). TWIST2, ZEB1, and ZEB2 were found to be correlated with YTHDF3 ( $\mathrm{r}=-0.156, \mathrm{P}=0.004 ; \mathrm{r}=0.243, \mathrm{P}=0.0016$; and $\mathrm{r}=0.231, \mathrm{P}=0.0027$, respectively). Subsequently, YTHDF3 was stably knocked down in the MDA-MB-231 and BT549 cell lines and the expression of TWIST2, ZEB1, and ZEB2 was examined. Indeed, knockdown of YTHDF3 markedly decreased ZEB1 mRNA and protein expression in both cell lines (Figure 3G,H). Therefore, ZEB1 was identified as a downstream target for YTHDF3 in TNBC.

\section{YTHDF3 enbances ZEB1 mRNA stability by $m^{6} A$ modification}

Since YTHDF3 is an important $\mathrm{m}^{6} \mathrm{~A}$ reader, we investigated whether YTHDF3 could interact with ZEB1 mRNA in an $\mathrm{m}^{6} \mathrm{~A}$-dependent manner. The results of the RIP assay revealed that YTHDF3 could bind to ZEB1 mRNA in MDA-MB-231 and BT-549 TNBC cells (Figure 4A). In addition, the ZEB1 mRNA in TNBC was modified with a m6A modification (Figure 4B). The mRNA actinomycin stability analysis showed that YTHDF3 inhibition impaired the stability of the ZEB1 mRNA in both MDA-MB-231 and BT-549 cells (Figure 4C,4D). The above data confirmed that YTHDF3 could enhance ZEB1 mRNA stability in an $\mathrm{m}^{6} \mathrm{~A}$-dependent manner.

\section{The YTHDF3/ZEB1 axis is involved in the progression of TNBC}

To further evaluate the effects of the YTHDF3/ZEB1 axis 
Table 2 Univariate and multivariate cox proportional hazard model for DFS and OS in TNBC patients

\begin{tabular}{|c|c|c|c|c|c|c|c|c|}
\hline \multirow{2}{*}{ Variables } & \multicolumn{4}{|c|}{ Univariate analysis } & \multicolumn{4}{|c|}{ Multivariate analysis } \\
\hline & $\mathrm{HR}(95 \% \mathrm{Cl})$ & $\mathrm{P}^{\mathrm{a}}$ & $\mathrm{HR}(95 \% \mathrm{Cl})$ & $\mathrm{P}^{\mathrm{a}}$ & $\mathrm{HR}(95 \% \mathrm{Cl})$ & $P^{a}$ & $\mathrm{HR}(95 \% \mathrm{Cl})$ & $\mathrm{P}^{\mathrm{a}}$ \\
\hline \multicolumn{9}{|c|}{ Age (years) } \\
\hline$\leq 50$ & Reference & & Reference & & Reference & & & \\
\hline \multicolumn{9}{|c|}{ Tumor size } \\
\hline$\leq 2 \mathrm{~cm}$ & Reference & & Reference & & Reference & & Reference & \\
\hline$>2 \mathrm{~cm}$ & $1.97(1.12-3.46)$ & 0.019 & $2.09(1.01-4.32)$ & 0.046 & $2.08(1.17-3.71)$ & 0.013 & $2.07(1.01-4.27)$ & 0.049 \\
\hline \multicolumn{9}{|c|}{ Lymph nodes metastasis } \\
\hline \multicolumn{9}{|l|}{ Grade } \\
\hline$I+I I$ & Reference & & Reference & & Reference & & & \\
\hline III & $1.94(1.08-3.49)$ & 0.026 & $1.99(0.94-4.22)$ & 0.072 & $1.83(1.01-3.31)$ & 0.047 & & \\
\hline \multicolumn{9}{|c|}{ Lymphovascular invasion } \\
\hline No & Reference & & Reference & & Reference & & & \\
\hline Yes & $1.73(1.04-2.88)$ & 0.036 & $1.76(0.92-3.36)$ & 0.086 & $1.59(0.94-2.70)$ & 0.084 & & \\
\hline \multicolumn{9}{|c|}{ YTHDF3 expression } \\
\hline Low & Reference & & Reference & & Reference & & Reference & \\
\hline
\end{tabular}

on TNBC, functional rescue experiments were conducted with YTHDF3 shRNA and ZEB1 overexpression vectors in TNBC cells. Wound healing and transwell assays revealed that the reduced migration and invasion ability induced by YTHDF3 inhibition could be recovered by ZEB1 overexpression (Figure $5 A, 5 B$ ). Moreover, the overexpression of ZEB1 also caused the downregulation of E-cadherin, as well as the upregulation of $\mathrm{N}$-cadherin and vimentin in YTHDF3 knockdown cells (Figure 5C). IHC staining was performed to assess the relationship between ZEB1 and YTHDF3 through Spearman's correlation analysis. As shown in Figure 5D,5E, the protein levels of ZEB1 were positively correlated with that of YTHDF3 in TNBC tumor tissues. Survival curve analysis also identified that patients with high ZEB1 expression were associated with poorer DFS and OS compared with patients with low
ZEB1 expression (Figure 5F). Moreover, a significantly poorer prognosis was found in TNBC patients with high expression levels of both YTHDF3 and ZEB1 (Figure 5G). Collectively, these findings suggested that ZEB1 is crucial for YTHDF3-mediated TNBC migration, invasion, and EMT. Indeed, the YTHDF3/ZEB1 axis is important in the progression of TNBC.

\section{Discussion}

TNBC is the most invasive subtype of BC with unfavorable outcomes. The lack of ER, PR, and HER2 expression renders TNBC patients inaccessible to endocrine or anti-HER2 target therapy. Currently, with no effective and clinically applicable therapeutic targets, the most common treatment strategy for TNBC is a combination of 
A

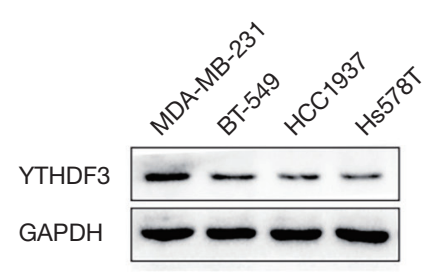

B

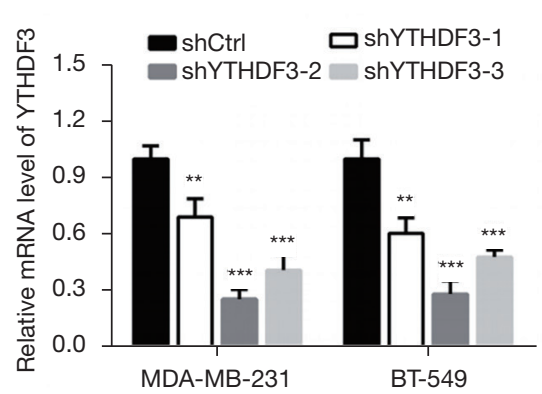

C

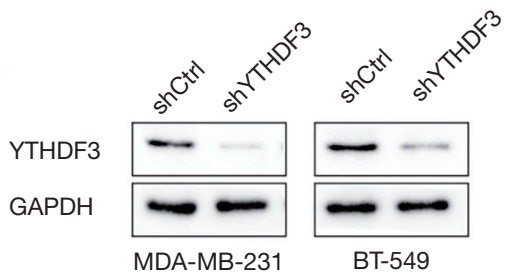

D
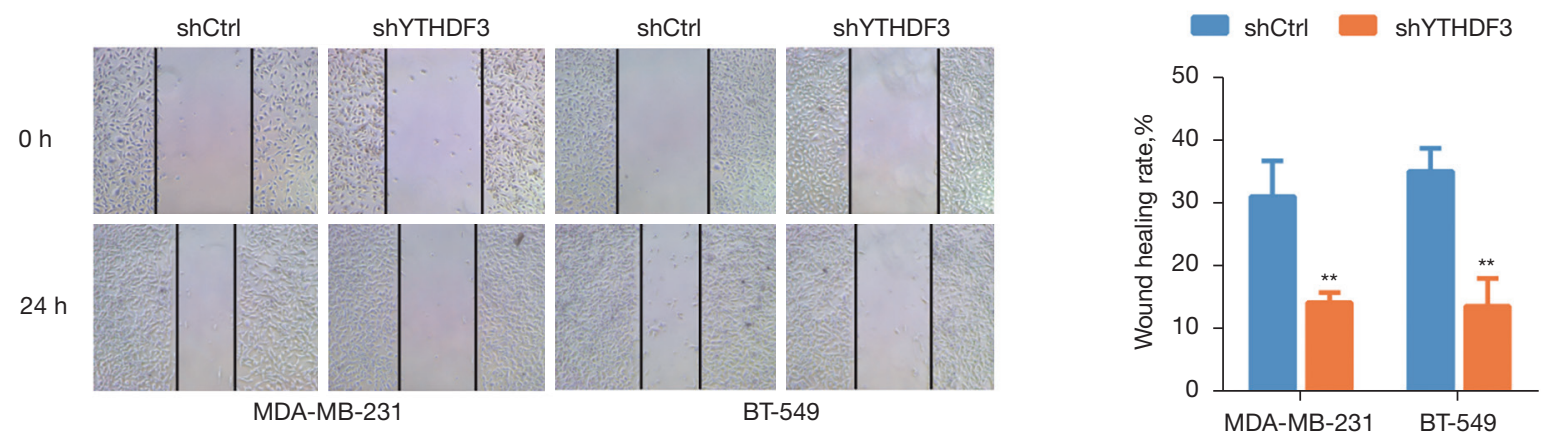

E
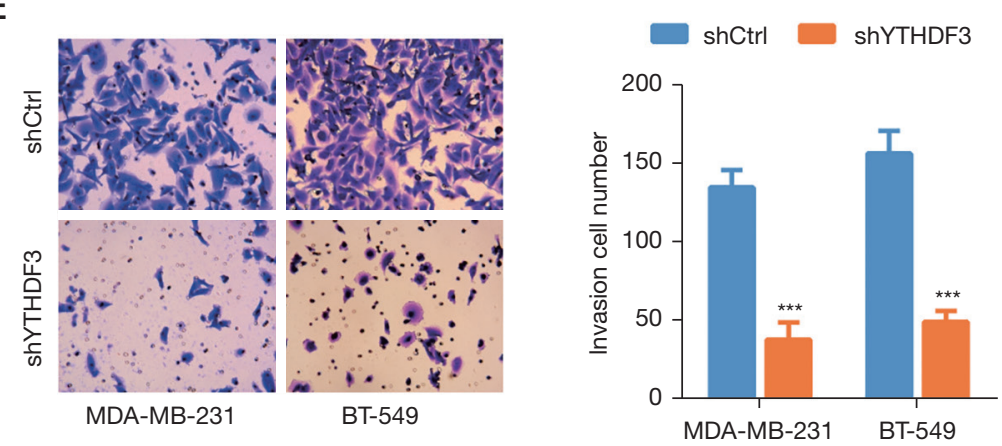

$\mathrm{F}$

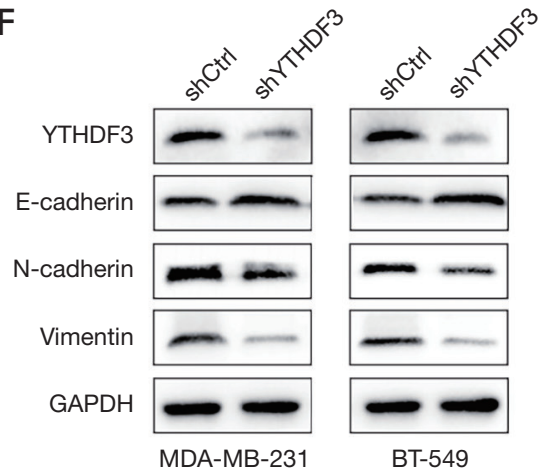

Figure 2 Knockdown of YTHDF3 suppresses cell migration, invasion, and EMT in MDA-MB-231 and BT-549 TNBC cells. (A) The protein expression of YTHDF3 in the MDA-MB-231, BT-549, HCC1937, and HS578T TNBC cell lines was detected by Western blot. (B,C) The knockdown efficiency of shYTHDF3 was analyzed by qRT-PCR and Western blot in MDA-MB-231 and BT-549 cells. (D,E) Cell migration and invasion ability were measured using the wound healing assay and transwell assay with YTHDF3 inhibition in MDA-MB-231 and BT-549 cells (transwell assay with crystal violet staining, $\times 200$ magnification). (F) The protein levels of the EMT markers (E-cadherin, $\mathrm{N}$-cadherin, and vimentin) were detected by Western blot with YTHDF3 knockdown in MDA-MB-231 and BT-549 cells. **, P<0.01; ***, $\mathrm{P}<0.001$. YTHDF3, the YTH domain family 3; GAPDH, glyceraldehyde 3-phosphate dehydrogenase; EMT, endothelial mesenchymal transition; TNBC, triple-negative breast cancer; qRT-PCR, quantitative real time-polymerase chain reaction.

surgery and adjuvant/neoadjuvant chemotherapy (28-30). Therefore, identification of novel regulatory molecules and promising therapeutic targets is beneficial to developing new treatment strategies for patients with TNBC.

$\mathrm{m}^{6} \mathrm{~A}$ is the most common post-transcriptional modification of RNA in eukaryotes, and has been identified to play key roles in various types of tumors $(9,10)$.
YTHDF3 is a crucial $\mathrm{m}^{6} \mathrm{~A}$ reader and is associated with the progression of gastric and colorectal cancer $(17,18)$. Previous studies have also demonstrated that YTHDF3 can induce brain metastasis in $\mathrm{BC}$ patients by enhancing the translation of $\mathrm{m}^{6} \mathrm{~A}$-enriched transcripts for ST6GALNAC5, gap junction alpha-1 protein (GJA1), and EGFR (19). However, there is a paucity of data regarding the function of 

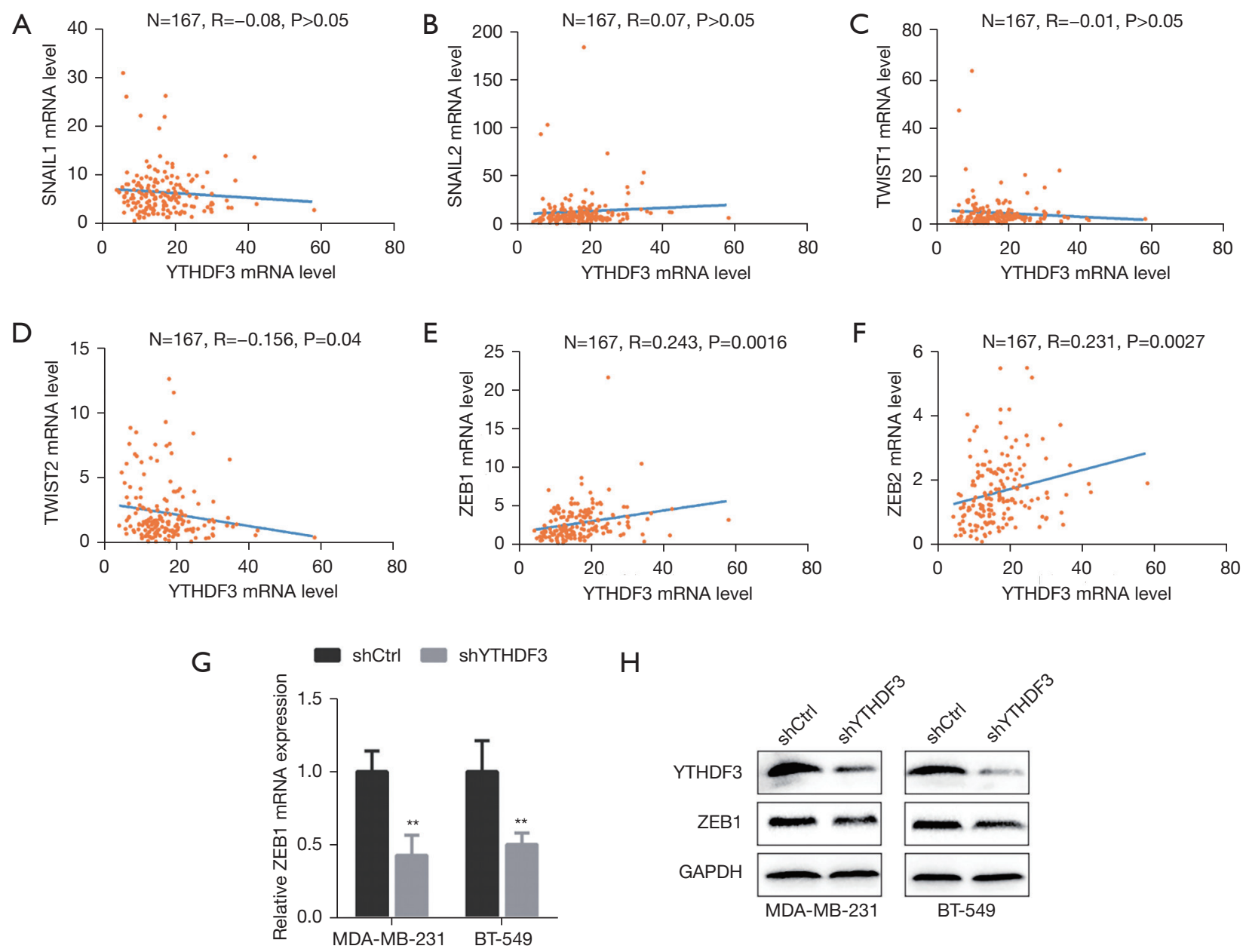

Figure 3 ZEB1 is a key downstream target for YTHDF3 in triple-negative breast cancer. (A-F) The correlation between YTHDF3 and EMT-TFs, SNAIL1, SNAIL2, TWIST1, TWIST2, ZEB1, and ZEB2, was evaluated using Spearman's correlation analysis based on TCGA database. (G) The mRNA expression of ZEB1 was identified by qRT-PCR after YTHDF3 knockdown in MDA-MB-231 and BT-549 cells. (H) The protein expression of ZEB1 was identified by Western blot after YTHDF3 knockdown in MDA-MB-231 and BT-549 cells. **, $\mathrm{P}<0.01$. YTHDF3, the YTH domain family 3; SNAIL1/2, snail family transcriptional repressor 1/2; TWIST1/2, twist family transcription factor 1/2; ZEB1/2, zinc finger E-box-binding homeobox 1/2; EMT-TF, endothelial mesenchymal transition transcription factor. TCGA, The Cancer Genome Atlas; qRT-PCR, quantitative real time-polymerase chain reaction.

YTHDF3 in TNBC. In this current study, the association of YTHDF3 expression with patient clinicopathological parameters and outcomes was evaluated using the TCGA database, the BC-GenExMiner tool, and IHC staining. The increased protein expression of YTHDF3 was closely related to more advanced histological grade and greater probability of lymph node metastasis. In addition, higher mRNA and protein expression of YTHDF3 was correlated with shortened survival for patients with TNBC. The above results collectively indicated that YTHDF3 may act as a promising biomarker for the prognosis of TNBC patients.
To further determine the function role of YTHDF3 in TNBC progression, a series of in vitro experiments were performed. Knockdown of YTHDF3 dramatically reduced the migration and invasion abilities of TNBC cells compared with the shRNA control group. It has been well demonstrated that EMT plays an important role in tumor progression and metastasis $(31,32)$. The downregulation of epithelial markers such as E-cadherin and the upregulation of mesenchymal markers such as $\mathrm{N}$-cadherin and vimentin are common hallmarks of EMT (24). Numerous studies have indicated that several $\mathrm{m}^{6} \mathrm{~A}$ regulators, 

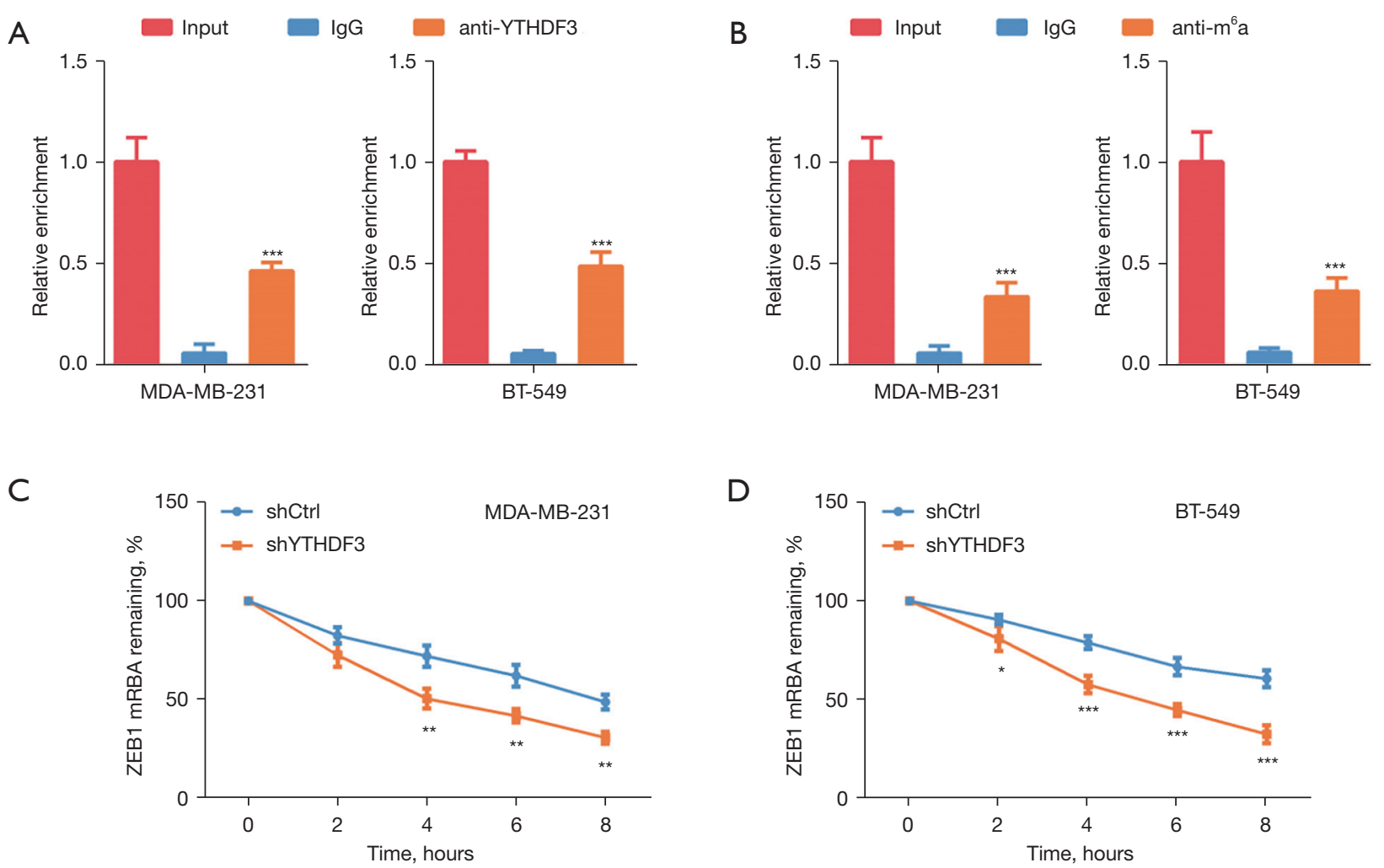

Figure 4 YTHDF3 enhances ZEB1 mRNA stability in an $\mathrm{m}^{6} \mathrm{~A}$-dependent manner. (A) RNA-binding protein immunoprecipitation (RIP) assay confirmed that Y'THDF3 can bind to ZEB1 mRNA in MDA-MB-231 and BT-549 cells. (B) RIP assay validated that ZEB1 mRNA was modified with $\mathrm{m}^{6} \mathrm{~A}$ modification in MDA-MB-231 and BT-549 cells. (C,D) The mRNA stability analysis verified that ZEB1 mRNA was drastically decreased after YTHDF3 knockdown in MDA-MB-231 and BT-549 cells. *, P<0.05; ${ }^{* *}, \mathrm{P}<0.01$; ${ }^{* * *}, \mathrm{P}<0.001$. YTHDF3, the YTH domain family 3; ZEB1, zinc finger E-box-binding homeobox 1; m² A, N6-methyladenosine.

including METTL3, METTL14, FTO, YTHDF1, and HNRNPA2B1, can promote tumor metastasis with an EMT-feature (33-38). Our study also revealed that YTHDF3 plays a critical role in EMT for TNBC. The expression levels of E-cadherin were upregulated, while $\mathrm{N}$-cadherin and vimentin expression were downregulated following the inhibition of YTHDF3. The correlation between YTHDF3 and EMT-TFs (SNAIL, TWIST, and ZEB families) was examined using TNBC samples in TCGA database. Interestingly, TWIST2, ZEB1, and ZEB2 were the 3 EMT-TFs found to be correlated with YTHDF3. Subsequent qRT-PCR and WB experiments confirmed that only ZEB1 mRNA and protein expression were markedly decreased in MDA-MB-231 and BT-549 cell lines. All these data confirmed that ZEB1 may be a downstream target for YTHDF3 in TNBC.

ZEB1 protein is a key transcriptional factor that promotes cellular plasticity and EMT in tumor cells
$(39,40)$. It has been reported to induce BC chemotherapy resistance and can transform non-invasive $\mathrm{BC}$ cells into highly malignant cancer stem cells (CSCs) (41-44). Since YTHDF3 is an important $\mathrm{m}^{6} \mathrm{~A}$ reader and previous studies have demonstrated that it can enhance ZEB1 mRNA stability via an $\mathrm{m}^{6} \mathrm{~A}$-dependent manner in hepatocellular carcinoma (45), the YTDF3-ZEB1 interaction was further investigated using RIP assays. The relevant results indicated that YTHDF3 can bind to ZEB1 mRNA in TNBC cells and ZEB1 mRNA in TNBC can be modified with $\mathrm{m}^{6} \mathrm{~A}$ modification. In addition, mRNA actinomycin stability analysis also demonstrated that inhibition of YTHDF3 can significantly impaired the stability of ZEB1 mRNA in MDA-MB-231 and BT-549 cells, further confirming that YTHDF3 can enhance ZEB1 mRNA stability in an $\mathrm{m}^{6} \mathrm{~A}$ dependent manner.

Functional rescue experiments were performed to verify the effects of the YTHDF3/ZEB1 axis on TNBC. 

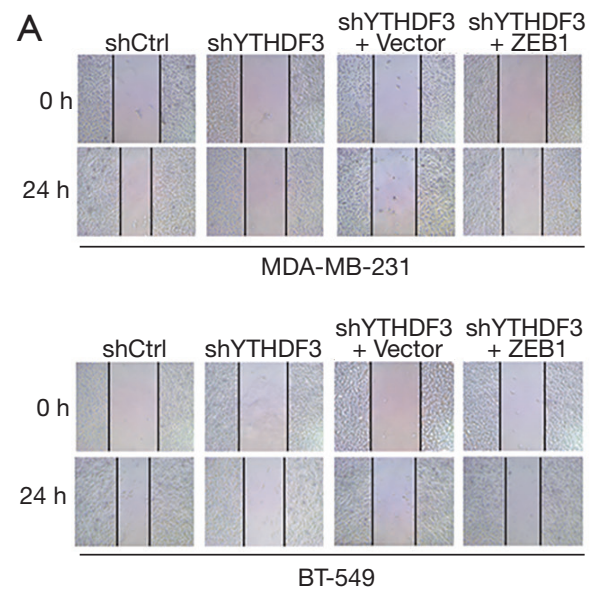

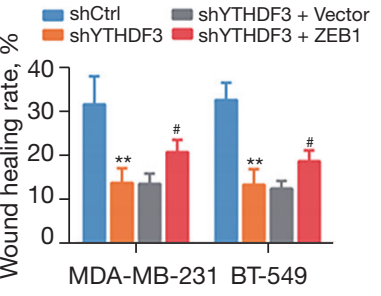

B

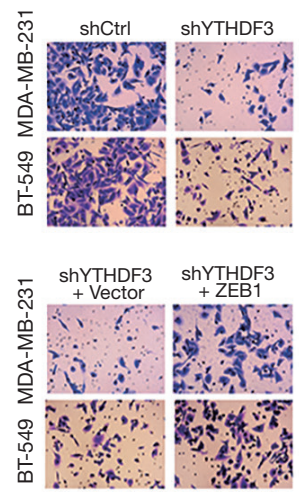

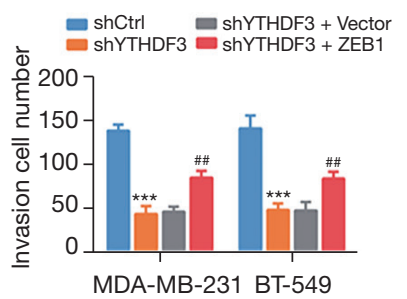
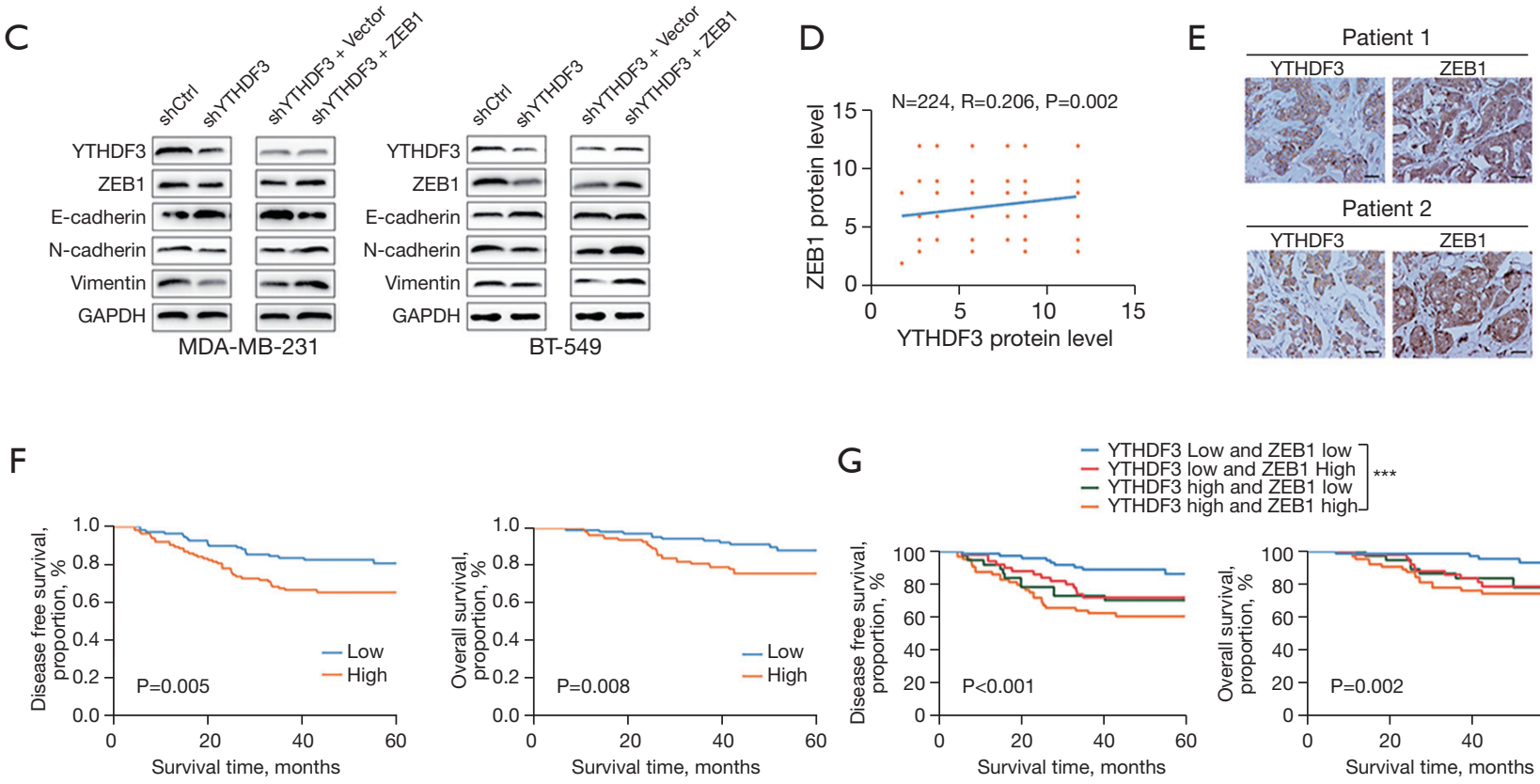

Figure 5 The YBX1/CTPS1 axis is involved in the progression of TNBC. (A,B) Cell migration and invasion ability were determined by wound healing and transwell assays after downregulation of YTHDF3 and overexpression of ZEB1 (transwell assay with crystal violet staining, $\times 200$ magnification). (C) The protein levels of the EMT markers (E-cadherin, N-cadherin, and vimentin) after downregulation of YTHDF3 and overexpression of ZEB1were detected by Western blot. (D) The correlation between YTHDF3 and ZEB1 protein expression was evaluated by Spearman's correlation analysis based on immunohistochemistry analysis. (E) Representative staining images of YTHDF3 and ZEB1 in TNBC tumor tissues (×200 magnification). (F) Kaplan-Meier analysis of the disease-free survival and overall survival with ZEB1 protein expression in TNBC patients. $(G)$ Kaplan-Meier analysis of the disease-free survival and overall survival in TNBC patients expressing both YTHDF3 and ZEB1. Scale bar: $50 \mu \mathrm{m}$. **, $\mathrm{P}<0.01$; ***, $\mathrm{P}<0.001$ (shCtrl vs. shYTHDF3); and * $\mathrm{P}<0.05$, \#\#, $\mathrm{P}<0.01$ (shYTHDF3 + Vector $v$ s. shYTHDF3 + ZEB1). YTHDF3, the YTH domain family 3; ZEB1, zinc finger E-box-binding homeobox 1; TNBC, triple-negative breast cancer; EMT, epithelial-mesenchymal transition.

The reduced migration and invasion ability induced by YTHDF3 inhibition could be recovered by ZEB1 overexpression. Moreover, the overexpression of ZEB1 also caused the downregulation of E-cadherin, and the upregulation of $\mathrm{N}$-cadherin and vimentin in YTHDF3 knockdown cells. The correlations between YTHDF3 and ZEB1 in TNBC patients was assessed by IHC staining of surgical specimens. The protein expression of YTHDF3 
was positively correlated with the expression of ZEB1. Patients with high ZEB1 expression endured a relatively poorer DFS and OS compared with patients with low ZEB1 expression. In addition, a significantly poorer survival was observed in patients with high expression levels of both YTHDF3 and ZEB1. Together, these results may represent a novel therapeutic target in the treatment of TNBC.

\section{Conclusions}

In summary, this investigation demonstrated that YTHDF3 is associated with poor prognosis for patients with TNBC and promotes the migration, invasion, and EMT of TNBC cells. ZEB1 was identified as a key downstream target for YTHDF3 and YTHDF3 can enhance ZEB1 mRNA stability in an $\mathrm{m}^{6} \mathrm{~A}$-dependent manner. Taken together, these findings suggest that YTHDF3 may be a critical prognostic factor and potential therapeutic target in TNBC.

\section{Acknowledgments}

Funding: This study was supported by grants from the Joint Funds for the Innovation of Science and Technology, Fujian Province (2018Y9205, 2019Y9054, 2019Y9103, 2020Y9053) and the Natural Science Foundation of Fujian Province (2021J01737).

\section{Footnote}

Reporting Checklist: The authors have completed the MDAR reporting checklist. Available at https://atm.amegroups. com/article/view/10.21037/atm-21-6857/rc

Data Sharing Statement: Available at https://atm.amegroups. com/article/view/10.21037/atm-21-6857/dss

Conflicts of Interest: All authors have completed the ICMJE uniform disclosure form (available at https://atm. amegroups.com/article/view/10.21037/atm-21-6857/coif). The authors have no conflicts of interest to declare.

Etbical Statement: The authors are accountable for all aspects of the work in ensuring that questions related to the accuracy or integrity of any part of the work are appropriately investigated and resolved. This study was approved by the Research Ethics Committee of Fujian Medical University Union Hospital (No. 2021KJCX039). All patients agreed to the use of their specimens and signed the informed consent forms. All procedures performed in this study involving human participants were in accordance with the Declaration of Helsinki (as revised in 2013).

Open Access Statement: This is an Open Access article distributed in accordance with the Creative Commons Attribution-NonCommercial-NoDerivs 4.0 International License (CC BY-NC-ND 4.0), which permits the noncommercial replication and distribution of the article with the strict proviso that no changes or edits are made and the original work is properly cited (including links to both the formal publication through the relevant DOI and the license). See: https://creativecommons.org/licenses/by-nc-nd/4.0/.

\section{References}

1. Sung H, Ferlay J, Siegel RL, et al. Global Cancer Statistics 2020: GLOBOCAN Estimates of Incidence and Mortality Worldwide for 36 Cancers in 185 Countries. CA Cancer J Clin 2021;71:209-49.

2. Foulkes WD, Smith IE, Reis-Filho JS. Triple-negative breast cancer. N Engl J Med 2010;363:1938-48.

3. Zhao S, Zuo WJ, Shao ZM, et al. Molecular subtypes and precision treatment of triple-negative breast cancer. Ann Transl Med 2020;8:499.

4. Bianchini G, Balko JM, Mayer IA, et al. Triplenegative breast cancer: challenges and opportunities of a heterogeneous disease. Nat Rev Clin Oncol 2016;13:674-90.

5. Roundtree IA, Evans ME, Pan T, et al. Dynamic RNA Modifications in Gene Expression Regulation. Cell 2017;169:1187-200.

6. Lin S, Choe J, Du P, et al. The m(6)A Methyltransferase METTL3 Promotes Translation in Human Cancer Cells. Mol Cell 2016;62:335-45.

7. Wang X, Zhao BS, Roundtree IA, et al. N(6)methyladenosine Modulates Messenger RNA Translation Efficiency. Cell 2015;161:1388-99.

8. Wang X, Lu Z, Gomez A, et al. N6-methyladenosinedependent regulation of messenger RNA stability. Nature 2014;505:117-20.

9. Lan Q, Liu PY, Haase J, et al. The Critical Role of RNA m6A Methylation in Cancer. Cancer Res 2019;79:1285-92.

10. Chen XY, Zhang J, Zhu JS. The role of m6A RNA methylation in human cancer. Mol Cancer 2019;18:103.

11. Xie J, Ba J, Zhang $M$, et al. The m6A methyltransferase METTL3 promotes the stemness and malignant progression of breast cancer by mediating $\mathrm{m} 6 \mathrm{~A}$ 
modification on SOX2. J BUON 2021;26:444-9.

12. Chen F, Chen Z, Guan T, et al. N6 -Methyladenosine Regulates mRNA Stability and Translation Efficiency of KRT7 to Promote Breast Cancer Lung Metastasis. Cancer Res 2021;81:2847-60.

13. Niu Y, Lin Z, Wan A, et al. RNA N6-methyladenosine demethylase FTO promotes breast tumor progression through inhibiting BNIP3. Mol Cancer 2019;18:46.

14. Petri BJ, Piell KM, South Whitt GC, et al. HNRNPA2B1 regulates tamoxifen- and fulvestrant-sensitivity and hallmarks of endocrine resistance in breast cancer cells. Cancer Lett 2021;518:152-68.

15. Shi H, Wang X, Lu Z, et al. YTHDF3 facilitates translation and decay of N6-methyladenosine-modified RNA. Cell Res 2017;27:315-28.

16. Li A, Chen YS, Ping XL, et al. Cytoplasmic m6A reader YTHDF3 promotes mRNA translation. Cell Res 2017;27:444-7.

17. Jing JJ, Zhao X, Li H, et al. Expression profiles and prognostic roles of m6A writers, erasers and readers in gastric cancer. Future Oncol 2021;17:2605-20.

18. Ni W, Yao S, Zhou Y, et al. Long noncoding RNA GAS5 inhibits progression of colorectal cancer by interacting with and triggering YAP phosphorylation and degradation and is negatively regulated by the m6A reader YTHDF3. Mol Cancer 2019;18:143.

19. Chang G, Shi L, Ye Y, et al. YTHDF3 Induces the Translation of m6A-Enriched Gene Transcripts to Promote Breast Cancer Brain Metastasis. Cancer Cell 2020;38:857-871.e7.

20. Anita R, Paramasivam A, Priyadharsini JV, et al. The m6A readers YTHDF1 and Y'THDF3 aberrations associated with metastasis and predict poor prognosis in breast cancer patients. Am J Cancer Res 2020;10:2546-54.

21. Lin Y, Lin L, Fu F, et al. Quantitative proteomics reveals stage-specific protein regulation of triple negative breast cancer. Breast Cancer Res Treat 2021;185:39-52.

22. Jézéquel P, Campone M, Gouraud W, et al. bcGenExMiner: an easy-to-use online platform for gene prognostic analyses in breast cancer. Breast Cancer Res Treat 2012;131:765-75.

23. van Staalduinen J, Baker D, Ten Dijke P, et al. Epithelialmesenchymal-transition-inducing transcription factors: new targets for tackling chemoresistance in cancer? Oncogene 2018;37:6195-211.

24. Wang Y, Shi J, Chai K, et al. The Role of Snail in EMT and Tumorigenesis. Curr Cancer Drug Targets 2013;13:963-72.
25. Caramel J, Ligier M, Puisieux A. Pleiotropic Roles for ZEB1 in Cancer. Cancer Res 2018;78:30-5.

26. Fardi M, Alivand M, Baradaran B, et al. The crucial role of ZEB2: From development to epithelial-to-mesenchymal transition and cancer complexity. J Cell Physiol 2019. [Epub ahead of print].

27. Budd GT, Barlow WE, Moore HC, et al. SWOG S0221: a phase III trial comparing chemotherapy schedules in highrisk early-stage breast cancer. J Clin Oncol 2015;33:58-64.

28. Early Breast Cancer Trialists' Collaborative Group (EBCTCG). Increasing the dose intensity of chemotherapy by more frequent administration or sequential scheduling: a patient-level meta-analysis of 37298 women with early breast cancer in 26 randomised trials. Lancet 2019;393:1440-52.

29. Loibl S, Poortmans P, Morrow M, et al. Breast cancer. Lancet 2021;397:1750-69.

30. Pastushenko I, Blanpain C. EMT Transition States during Tumor Progression and Metastasis. Trends Cell Biol 2019;29:212-26.

31. Lambert AW, Pattabiraman DR, Weinberg RA. Emerging Biological Principles of Metastasis. Cell 2017;168:670-91.

32. Serrano-Gomez SJ, Maziveyi M, Alahari SK. Regulation of epithelial-mesenchymal transition through epigenetic and post-translational modifications. Mol Cancer 2016;15:18.

33. Zhao C, Ling X, Xia Y, et al. The m6A methyltransferase METTL3 controls epithelial-mesenchymal transition, migration and invasion of breast cancer through the MALAT1/miR-26b/HMGA2 axis. Cancer Cell Int 2021;21:441.

34. Liang $X$, Zhang Z, Wang L, et al. Mechanism of methyltransferase like 3 in epithelial-mesenchymal transition process, invasion, and metastasis in esophageal cancer. Bioengineered 2021;12:10023-36.

35. Shi Y, Zhuang Y, Zhang J, et al. METTL14 Inhibits Hepatocellular Carcinoma Metastasis Through Regulating EGFR/PI3K/AKT Signaling Pathway in an m6A-Dependent Manner. Cancer Manag Res 2020;12:13173-84.

36. Shimura T, Kandimalla R, Okugawa Y, et al. Novel evidence for m6A methylation regulators as prognostic biomarkers and FTO as a potential therapeutic target in gastric cancer. Br J Cancer 2022;126:228-37.

37. Bian $\mathrm{S}, \mathrm{Ni} W$, Zhu M, et al. Identification and Validation of the N6-Methyladenosine RNA Methylation Regulator YTHDF1 as a Novel Prognostic Marker and Potential Target for Hepatocellular Carcinoma. Front Mol Biosci 2020;7:604766. 
38. Zhu F, Yang T, Yao M, et al. HNRNPA2B1, as a m6A Reader, Promotes Tumorigenesis and Metastasis of Oral Squamous Cell Carcinoma. Front Oncol 2021;11:716921.

39. Krebs AM, Mitschke J, Lasierra Losada M, et al. The EMT-activator Zeb1 is a key factor for cell plasticity and promotes metastasis in pancreatic cancer. Nat Cell Biol 2017;19:518-29.

40. Chaffer CL, Marjanovic ND, Lee T, et al. Poised chromatin at the ZEB1 promoter enables breast cancer cell plasticity and enhances tumorigenicity. Cell 2013;154:61-74.

41. Zhang X, Zhang Z, Zhang Q, et al. ZEB1 confers chemotherapeutic resistance to breast cancer by activating ATM. Cell Death Dis 2018;9:57.

42. Wellner U, Schubert J, Burk UC, et al. The EMT- activator ZEB1 promotes tumorigenicity by repressing stemness-inhibiting microRNAs. Nat Cell Biol 2009;11:1487-95.

43. Luo H, Zhou Z, Huang S, et al. CHFR regulates chemoresistance in triple-negative breast cancer through destabilizing ZEB1. Cell Death Dis 2021;12:820.

44. Wang G, Dong Y, Liu H, et al. Loss of miR-873 contributes to gemcitabine resistance in triple-negative breast cancer via targeting ZEB1. Oncol Lett 2019;18:3837-44.

45. Wang M, Yang Y, Yang J, et al. circ_KIAA1429 accelerates hepatocellular carcinoma advancement through the mechanism of m6A-YTHDF3-Zeb1. Life Sci 2020;257:118082.

(English Language Editor: J. Teoh)
Cite this article as: Lin $Y$, Jin $X$, Nie Q, Chen M, Guo W, Chen L, Li Y, Chen X, Zhang W, Chen H, Jiang M, Xiao H, Zhang J, Fu F, Wang C. YTHDF3 facilitates triple-negative breast cancer progression and metastasis by stabilizing ZEB1 mRNA in an $\mathrm{m}^{6} \mathrm{~A}$-dependent manner. Ann Transl Med 2022;10(2):83. doi: 10.21037/atm-21-6857 
Supplementary

Table S1 A list of shRNA sequence and primers used in this study

\begin{tabular}{lcl}
\hline Application & Name & Sequence \\
\hline shRNA & shYTHDF3-1 & 5'-GGATCTCAGGGACAATCAACA-3' \\
& shYTHDF3-2 & 5'-GCCTCAGCCATTAATTCAACC-3' \\
primers for qRT-PCR & shYTHDF3-3 & 5'-GCATACCACCTCAATCTTTGA-3' \\
& YTHDF3-F & 5'-TGTTGTGGACTATAATGCGTATGC-3' \\
& YTHDF3-R & 5'-AAGCGAATATGCCGTAATTGGTTA-3' \\
& TWIST2-F & 5'-CAGAGCGACGAGATGGACAAT-3' \\
& TWIST2-R & 5'-TAGTGGGAGGCGGACATGGA-3' \\
ZEB1-F & 5'-CAGCTTGATACCTGTGAATGGG-3' \\
ZEB1-R & 5'-TATCTGTGGTCGTGTGGGACT-3' \\
ZEB2-F & 5'-GATATGACAGACTCCGACTCCT-3' \\
ZEB2-R & 5'-CGATAAGGTGGTGCTTGTGTT-3' \\
GAPDH-F & 5'-TGACTTCAACAGCGACACCCA-3' \\
GAPDH-R & 5'-CACCCTGTTGCTGTAGCCAAA-3'
\end{tabular}

qRT-PCR, quantitative real time-polymerase chain reaction. 